\title{
Formation energy and interaction of point defects in two-dimensional colloidal crystals
}

\author{
L.C. DaSilva $\stackrel{\dagger}{\dagger}$, L. Cândido \\ ${ }^{\dagger}$ Instituto de Física de São Carlos, Universidade de São Paulo 13560-970, São Carlos, SP, Brazil \\ ${ }^{\ddagger}$ Instituto de Física, Universidade Federal de Goiás, Campus II , 74001-970 Goiânia, GO, Brazil
}

\begin{abstract}
The manipulation of individual colloidal particles using optical tweezers has allowed vacancies to be created in two-dimensional (2d) colloidal crystals, with unprecedented possibility of real-time monitoring the dynamics of such defects (Nature 413, 147 (2001)). In this Letter, we employ molecular dynamics (MD) simulations to calculate the formation energy of single defects and the binding energy between pairs of defects in a $2 \mathrm{~d}$ colloidal crystal. In the light of our results, experimental observations of vacancies could be explained and then compared to simulation results for the interstitial defects. We see a remarkable similarity between our results for a $2 \mathrm{~d}$ colloidal crystal and the 2d Wigner crystal (Phys. Rev. Lett. 86, 492 (2001)). The results show that the formation energy to create a single interstitial is $12 \%-28 \%$ lower than that of the vacancy. Because the pair binding energies of the defects are strongly attractive for short distances, the ground state should correspond to bound pairs with the interstitial bound pairs being the most probable.
\end{abstract}

\section{INTRODUCTION}

A revival of general interest in point defects in twodimensional (2d) systems has been fueled by demonstration that vacancies can be created in $2 \mathrm{~d}$ colloidal crystals through manipulation of individual particles with optical tweezers 1.2 . Because of the large size of the colloidal particles, the structural and dynamical properties of these point defects can be monitored with video microscopy ${ }^{3}$. Interest in point defects in solids is widespread, including defects in ordinary materials as well as in quantum crystals such as ${ }^{4} \mathrm{He}$ and Wigner crystals. In quantum crystals the point defects are believed to occur at finite concentrations at any nonzero temperature. There is also speculation that even at zero temperature point defects can exist, while at higher concentrations they may lead to a supersolid phase ${ }^{4,5.8}$. The role of point defects in melting in a two-dimensional system has been investigated theoretically $\frac{6,7,9,10}{}$, but an experimental investigation is usually hampered by the low concentrations of defects, unless the temperature is close to the melting point ${ }^{11}$.

In this work, we report on the first accurate Molecular Dynamics (MD) calculations of the formation energy of single point defects and interaction of pair point defects in a $2 \mathrm{~d}$ screened Coulomb interaction colloidal system. The system is formed by colloidal particles, taken as identical spheres of radius $a$, suspended in a solvent (generally water) and confined between two parallel solid surfaces. When immersed in this solvent, the colloids acquire a large charge $Z$ due to dissociation of endgroups from their surface. Counterions thus generated ensure charge neutrality, forming a cloud around each colloid that makes the Coulomb interaction shorter. The colloids are free to move in $2 \mathrm{~d}$ and interact through pairwise Yukawa type potential ${ }^{12,13}$. The Hamiltonian for this system is

$$
H=\sum_{i}^{N} \frac{\mathbf{p}_{\mathbf{i}}^{2}}{2 m}+\sum_{i<j}^{N} \frac{\left(Z_{\lambda} e\right)^{2}}{\epsilon} \frac{e^{-\mathbf{r}_{\mathbf{i j}} / \lambda}}{\mathbf{r}_{\mathbf{i j}}}+N U_{B},
$$

where the first term in the right is the kinetic energies, the second is the screened Coulomb colloid-colloid interaction and the third term is the interaction between the colloids and the neutralizing background of positive charges ${ }^{14} U_{B}=-2 \pi b \lambda / a_{0}^{2}$, where $b=(2 / \sqrt{3})^{1 / 2} a_{0}$ is the lattice space and $a_{0}$ is the average separation between colloids (this is defined for the triangular lattice with vector translation $(b, 0)$ and $(b / 2, b \sqrt{3} / 2)$ with $a_{0}=1 / \sqrt{\rho}$ where $\rho$ is the $2 \mathrm{~d}$ colloid number density), $\lambda$ is the screening length, $\epsilon$ is the dielectric constant of the medium and $Z_{\lambda}=Z^{*} f(a / \lambda)$, where $Z^{*}$ is the normalized charge and $f(x)=\sinh (x) / x$ is a function that describes the effect of the nonzero radius $a$ of the colloidal particles. The energy, length, temperature and time are in units of $E_{0}=\left(Z_{\lambda} e\right)^{2} / \epsilon \sigma$, where $\sigma=1.1 \mu \mathrm{m}$ (typical lattice space for experimental systems), $T_{0}=E_{0} / \kappa_{B}\left(\kappa_{B}\right.$ Boltzmann constant) and $t_{0}=\left(E_{0} / m \sigma^{2}\right)^{-1 / 2}$, respectively.

\section{COMPUTATIONAL DETAILS}

The system with point defects (vacancy or interstitial) is modeled by removing (adding) a colloidal particle from (to) the most stable $2 \mathrm{~d}$ lattice, which is the triangular lattice. We place the defect at the center of the simulation box to avoid complications arising from lattice relaxation: a single 6 coordinated vacancy at the central lattice site or a threefold centered interstitial colloid in one of the central triangular unit cells. However, no constraint exists which would restrict the center of each colloid to lie within its own Wigner-Seitz cell, i.e. to make the lattice relaxation locally. This means that the defects are free to move around and can change their symmetry during the system evolution in thermodynamic equilibrium. 
In order to calculate the energy needed to create one single defect we perform two independent simulations at the same density and temperature: a simulation for the perfect and a simulation for the defective system. For the latter, after introducing the defect, we rescale the dimensions of the simulation box by a factor $f=\sqrt{n_{d} / n_{p}}$ to reset the system to the original density, with $n_{d}$ and $n_{p}$ being the number of colloids for the defective and the perfect system, respectively. This is performed to circumvent the need of correcting the energy due to density change caused by inclusion of the defect. The difference between the energies of the defective and the perfect systems is the energy needed to create the defect. Formally, we can define the number of defects $N_{\text {def }}$ as the number of colloids minus the number of lattice sites. Therefore, the formation energy of $N_{\text {def }}$ defects in a crystal with $N$ lattice sites is

$$
\Delta E_{\text {def }}=\left[\tilde{E}\left(N+N_{\text {def }}\right)-\tilde{E}(N)\right]\left(N+N_{\text {def }}\right),
$$

where $\tilde{E}(n)$ is the energy per colloid for a system containing $n$ colloids. For a monovacancy or an interstitial defect, $N_{\text {def }}=N_{v}=-1$ and $N_{d e f}=N_{i n t}=+1$, respectively.

MD calculations of the formation energy of point defects were performed for several system sizes $n=29,30$, $31,129,130,131,269,270,271,479,480$ and 481 colloids, which allows one to study finite-size effects at different densities. Larger system sizes would be unpractical computationally because high accuracy is needed in order to obtain the energy differences.

We used colloids with radius $a \sim 0.18 \mu \mathrm{m}$, screening length $\kappa^{-1} \sim 0.39 \mu \mathrm{m}$, charge $\sim 1650 e$ and density varying from $\rho=0.402$ to $1.804(\mu \mathrm{m})^{-2}$, corresponding to typical experimental data $\stackrel{1}{\text {. To }}$ To give an idea of the energies and temperatures involved in the calculations, for instance at $\rho=0.954(\mu \mathrm{m})^{-2}$ the units are $E_{0}=9.4 \times 10^{-18}$ Joules and $T_{0}=6.81 \times 10^{5} \mathrm{~K}$.

The initial positions for the colloids are the sites of a triangular lattice accommodated in a rectangular box with periodic boundary conditions to eliminate surface effects. The simulations were performed within the canonical ensemble keeping a constant system temperature using the Berendsen's thermostat with coupling parameter to an external bath $\tau_{T}$ ranging from 0.01 to $0.1 \frac{16}{}$. The evolution of Newton's equation of motion is obtained with the four-order predictor-corrector algorithm. The time step varies from $2.5 \times 10^{-2}$ to $5.0 \times 10^{-3} t_{0}$ since it has some scale dependence on the colloid density. Thermodynamic equilibrium was assumed to be achieved during the first 50000 time steps, after which the physical quantities were obtained by averaging over 700 blocks of 10000 time steps.

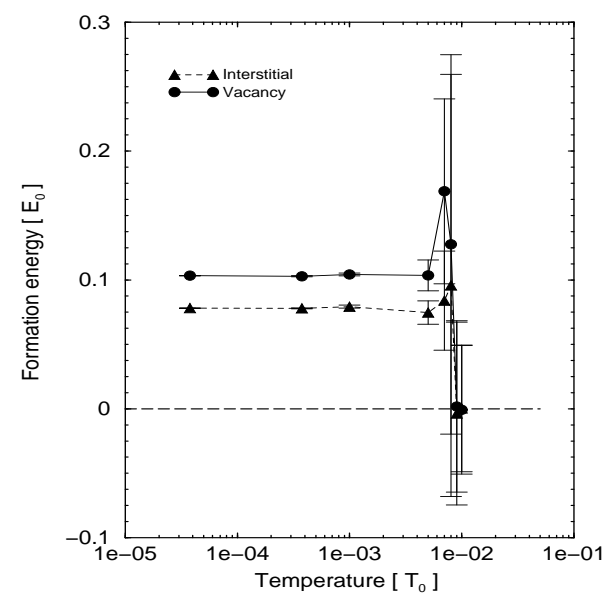

FIG. 1: Formation energy of a vacancy and interstitial defect as a function of temperature at $\rho=0.954(\mu \mathrm{m})^{-2}$. Computed for systems of 120 lattice sites.

\section{RESULTS AND DISCUSSION}

In the simulation, the formation energy of the vacancy and interstitial defects depends on the number of particles, i.e. size of the simulation box. To eliminate the size dependence of the formation energy we extrapolate the simulation results to the thermodynamic limit by using the following formula

$$
\Delta E_{d e f}^{N}(\rho)=\Delta E_{d e f}^{\infty}(\rho)+\frac{c(\rho)}{N},
$$

where the density-dependent parameters $\Delta E_{\text {def }}^{\infty}(\rho)$ and $c(\rho)$ are determined by a linear least-squares fit to the MD calculations at different $N$. The MD formation energies for the point defects and $\chi^{2}$ of the fitted data as a function of $\rho$, at a fixed temperature and screening length, are shown in table I. The $\chi^{2}$ of the fitted data is particularly good, indicating that the size dependence is well described by Eq. (3). The formation energies for interstitial defects are consistently lower, from $12 \%-28 \%$, than those for vacancies in the range of densities studied. Therefore, an interstitial defect is more stable and more likely to be created.

The formation energy of the defects is practically temperature-independent in the temperature range $3 . \times$ $10^{-5} T_{0}<T<5 . \times 10^{-2} T_{0}$ below the melting point, as shown in Fig. 1, where the formation energy of the interstitials is smaller than that of the vacancies. However, when the kinetic energy starts to compete with the potential energy close to the melting point $\left(T>5.0 \times 10^{-2} T_{0}\right)$, the formation energy of the defects becomes too noisy due to large fluctuations in the total energy and approaches zero within the statistical error. This suggests that single point defects may be easily created thermally in this temperature range and could play an important role in the melting mechanisms of $2 \mathrm{~d}$ colloidal crystals. 
TABLE I: Formation energy in reduced units obtained from MD simulations for a vacancy (left) and interstitial defect (right) for various system sizes denoted by $N$ (number of lattice sites) and at various values of $\rho$ in $(\mu \mathrm{m})^{-2}$. Quantities in parentheses are the estimated error in the last decimal place. Also given are the energies for an infinite system $\left(\Delta E_{V a c}^{\infty}, \Delta E_{I n t}^{\infty}\right), \mathrm{c}(\rho)$ and $\chi^{2}$ fitting parameters. The system temperature and the screening length are $T=3.74 \times 10^{-4} T_{0}$ and $\kappa^{-1}=0.39 \mu$, respectively.

\begin{tabular}{ccccc||ccccc}
\hline \hline \multicolumn{5}{c||}{ Vacancy } & \multicolumn{5}{c}{ Interstitial } \\
& $\rho=0.402$ & $\rho=0.589$ & $\rho=0.954$ & $\rho=1.804$ & & $\rho=0.402$ & $\rho=0.589$ & $\rho=0.954$ & $\rho=1.804$ \\
\hline$N=30$ & $0.0261(5)$ & $0.0543(4)$ & $N=30$ & $0.0238(5)$ & $0.0430(4)$ \\
$N=120$ & $0.029(1)$ & $0.0550(4)$ & $0.1029(4)$ & $0.1933(4)$ & $N=120$ & $0.024(1)$ & $0.0435(6)$ & $0.0781(5)$ & $0.1425(3)$ \\
$N=270$ & $0.029(2)$ & $0.055(1)$ & $0.1032(4)$ & $0.1958(7)$ & $N=270$ & $0.024(2)$ & $0.043(3)$ & $0.0783(6)$ & $0.1426(4)$ \\
$N=480$ & $0.029(3)$ & $0.055(1)$ & $0.1032(9)$ & $0.1958(6)$ & $N=480$ & $0.024(2)$ & $0.043(6)$ & $0.0785(5)$ & $0.1428(5)$ \\
$\Delta E_{\text {Vac }}^{\infty}$ & $0.029(1)$ & $0.0551(4)$ & $0.1033(4)$ & $0.1969(7)$ & $\Delta E_{I n t}^{\infty}$ & $0.024(1)$ & $0.0436(7)$ & $0.0785(6)$ & $0.1428(5)$ \\
$\mathrm{c}(\rho)$ & $-0.10(3)$ & $-0.02(2)$ & $-0.05(8)$ & $-0.4(1)$ & $\mathrm{c}(\rho)$ & $-0.00(3)$ & $-0.01(3)$ & $-0.0(1)$ & $-0.03(8)$ \\
$\chi^{2}$ & 0.086 & 0.032 & 0.023 & 0.6 & $\chi^{2}$ & 0.00056 & 0.046 & 0.018 & 0.049 \\
\hline \hline
\end{tabular}

We now investigate the possible defect topologies as the system evolves in thermodynamic equilibrium, which is particularly relevant for defining the dynamics of the defects, as observed experimentally $\underline{\underline{1}}$ and recently corroborated by results from Brownian simulation methods $\frac{15}{}$. According to our simulations, the initial configurations for the threefold centered interstitial and the sixfold vacancy were found to relax into a configuration of lower symmetry, in agreement with simulation and experimental observations. However, the aforementioned observations concerning the dynamics of defects focus only on the symmetry and topology of the defect. In the following we discuss the dynamics of the defects in topological and energetic terms.

As stated in $\operatorname{Ref} \frac{3}{2}$, at finite temperatures this many body system vibrates around every local energy minimum due to thermal fluctuations. If the energy differences between distinct local minima are small, the system can get enough energy to move to a nearby local minimum. As long as the system remains around a local energy minimum, the distortions in the lattice are elastic and the topological arrangement of the particles does not change. This allows us to calculate the system energy for each topology. For such a calculation, the defect must be tracked after the system has reached thermodynamic equilibrium. For that, we developed a code to perform a dynamical check of a list of neighbors of each colloid in a triangular lattice. This list is created (updated) by counting the sides of the polygons in the Voronoi 17 construction at each time step run. A defect is characterized by the presence of miscoordinated particles, i.e. particles whose number of neighbors is different from 6 . Once the current topology of the defect is identified, the corresponding energy is recorded. We also calculated the time the defect remains in a given topology and the number of transitions each defect performs between different topological configurations.

Table II summarizes our findings for the dynamics of the defects. The topologies for the vacancy are: crushed vacancy $\left(V_{2}\right)$; symmetric vacancy $\left(V_{3}\right)$; split vacancy $(S V)$; which were observed experimentally $\underline{\underline{1}}$, and an- other one, a fourfold symmetric excited configuration $\left(V_{4}^{\prime}\right)$, only observed recently 15 . For interstitial defects the topologies are: threefold symmetric interstitial $\left(I_{3}\right)$; twofold symmetric interstitial $\left(I_{2}\right)$; disjoint twofold symmetric interstitial $\left(I_{2 d}\right)$; and a fourfold symmetric excited interstitial $\left(I_{4}^{\prime}\right)$. The transition matrix (a stochastic matrix) in the upper part of Table II indicates that for a sufficiently long time, both vacancy and interstitial defects adopt the possible topologies many times. Each row of this matrix gives the probability of transition from a state (topology) to another one. As pointed out in Ref. $\frac{15}{15}$, the topologies $V_{2}, S V, I_{2}$ and $I_{2 d}$ have a preferential diffusion direction, with motion being a random walk along the main crystalline directions. In contrast, the $V_{3}, V_{4}^{\prime}, I_{3}$ and $I_{4}^{\prime}$ have no preferential direction and may act in switching the direction of motion. Therefore, the diffusion process of our system, for a long time run, is isotropic. However, we observed in subsidiary simulations (results not shown here) that for short runs there is a very small number of transitions to topological configurations responsible for changing the direction of motion $\left(V_{3}, V_{4}^{\prime}, I_{3}\right.$ and $\left.I_{4}^{\prime}\right)$, especially for the vacancy. As a result, diffusion becomes one-dimensional, consistent with experimental data and simulation results ${ }^{1,15}$.

At the bottom of Table II, we show the times $\left(\tilde{t}_{s}\right)$ for the defects in each topological configuration in equilibrium, as well as the formation energy $\Delta E$ for each topological configuration. The formation energy for the various topologies are very close to each other, being almost within the statistical error. However, one infers that the lowest formation energy for both vacancy and interstitial defects correspond to the configurations $S_{V}$ and $I_{3}$, where in average the defects remain most of the time $\left(\tilde{t}_{s}\right)$. This result should be expected as these configurations are the most likely to be thermally activated since they need the smaller amount of energy to be created. On the other hand, the defect tends to spend a very short time in $V_{4}^{\prime}$ and $I_{4}^{\prime}$ configurations because they have the highest formation energies. Note that $I_{2 d}$ topology is just a transient variation of $I_{2}$ topology. A direct comparison of the formation energies for each topological configuration 


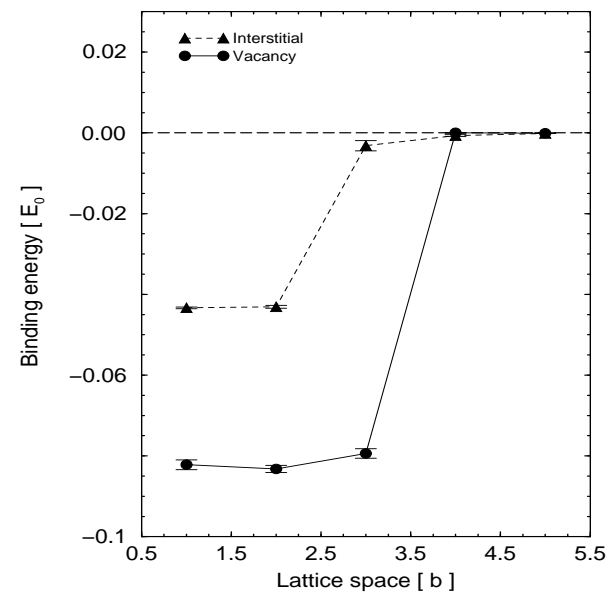

FIG. 2: Binding energy of a pair defect of vacancy-vacancy and interstitial-interstitial as a function of lattice spaces at $\rho=0.954(\mu \mathrm{m})^{-2}$. Computed for systems of 120 lattices sites.

and its lifetime can lead to wrong interpretation of the results of Table II. Although the defects formation energies between such topological configurations are similar, the average time of the defect in each topological configuration is quite different due to differences in the energy barriers. For instance, though the formation energy of $V_{2}$ is slightly smaller than that of $V_{3}$, the lifetime of $V_{2}$ is much larger. The reason is that the net transition probability from $V_{3}$ to $S V$ is much larger than that from $V_{2}$ to $S V$ (about, $36 \%$, see transition matrix), which indicates a smaller energy barrier between $V_{3}$ and $S V$ topologies making $V_{3}$ less stable than $V_{2}$. One can make also the same discussion for the interstitials defect (right hand side of the table II).

Far from the melting point, an increase in temperature causes the number of transitions to increase, including the low-frequency transitions between different topological configurations. Close to the melting transition, the formation energy for different topological configurations is difficult to calculate owing to the large thermal fluctuations, with the differences in energy lying within statistical error.

We also studied the interaction of a pair of defects as a function of the lattice separation. The difference between the energies to create two single defects separated by a given number of lattice constants and the energy to create two isolated single defects is the binding energy between two single defects, defined as follows

$$
E_{\text {int }}=\Delta E_{\text {def }}\left(N_{\text {def }}=2\right)-2 \Delta E_{\text {def }}\left(N_{\text {def }}=1\right)
$$

where the first term in the right $\Delta E_{\text {def }}\left(N_{\text {def }}=2\right)$ corresponds to the energy to create two single defects separated by some lattice constants, while the last term $2 \Delta E_{\text {def }}\left(N_{\text {def }}=1\right)$ is the energy to create two isolated single defects.
Fig. 2 shows the binding energy vs. lattice separation. In order to enforce accuracy in positioning the pairs of defects, we slow down the movement of the colloids by decreasing the temperature to $\sim 10^{-10} T_{0}$. It was enough to hold the two defects in a fixed separation. Both the pairs of vacancy-vacancy and interstitial-interstitial are strongly attractive at short distances, with attraction going to zero for distances greater than three lattice spaces. For short distances the binding energies for the defects are $\sim-0.08$ and $\sim-0.04$ for the vacancy and interstitial defect, respectively, being therefore higher than the value expected at the melting temperature at density $\rho=0.954(\mu \mathrm{m})^{-2}$, which is $\sim 0.01$ according to Fig. 1. We have also (not shown here) calculated the binding energy as a function of the density for just one lattice space separation between defects, which resulted always attractive in the range of densities $\rho=0.402-1.804$ $(\mu \mathrm{m})^{-2}$. Since any attraction should suffice to permit recombination, our results suggest that the ground state energy of the $2 \mathrm{~d}$ colloidal crystal may be dominated by pair binding of defects. Furthermore, as interstitial defects have the lowest excitation energy, we expect that the ground state of point defects should involve mainly interstitial pairs. Though the mechanisms of pair binding of point defects in solids are still not fully understood, we note that our results are similar to those for interstitial defects in quantum crystals, such as vortex crystals 9 and $2 \mathrm{~d}$ Wigner crystals 8 , in which the defect pair is not sufficiently strong to yield a supersolid phase. For experimentalists working in the $2 \mathrm{~d}$ colloidal system using video microscopy, we believe the results on the pair binding of defects provide an experimental challenge to observe formation of pair point defects near the $2 \mathrm{~d}$ colloidal crystal melting.

Before concluding, we comment on the entropic factors, which are embedded in the calculations of the formation energies. Such factors could be neglected well below the melting temperature, as they are much less important than the energy terms. Close to the melting temperature, entropic factors are likely to affect the numerical values of the formation energies, but the qualitative features should be preserved. Indeed, this expectation appears to be fulfilled in the experimental system represented by the $2 \mathrm{~d}$ colloidal crystal, since our simulations could explain the experimental observations.

In conclusion, we have shown quantitatively the effects from point defects (vacancy and interstitial) in $2 \mathrm{~d}$ colloidal crystals, in which the energy to create a single interstitial defect is $12 \%-28 \%$ lower than to create a single vacancy. The formation energies of these defects go to zero near the melting point, i.e. point defects can be easily created thermally and should play a crucial role in the melting mechanisms for such crystals. We also confirmed previous results that the interstitial defects are more mobile than vacancies, and provided an explanation based on energy calculations. Finally, we found that the interaction between defects is strongly attractive, and consequently most defects will exist as bound pairs. We believe 
TABLE II: The upper part is the normalized transition matrix for different topological configurations of the defects, during $\sim 7.0 \times 10^{6} \mathrm{MD}$ steps after the system has reached equilibrium. In the bottom are displayed the time spent in each topological configuration $\left(\tilde{t}_{s}\right)$ and the formation energy in reduced units of the defects, $\Delta E$, for the $2 \mathrm{~d}$ colloidal crystal at $\rho=0.954$ $(\mu \mathrm{m})^{-2}$. Quantities in parentheses are the estimated errors in the last decimal place. The system temperature and screening length are $T=1.0 \times 10^{-3} T_{0}$ and $\kappa^{-1}=0.39 \mu \mathrm{m}$, respectively.

\begin{tabular}{ccccc||ccccc}
\hline \hline \multicolumn{9}{c|}{ Vacancy } & \multicolumn{5}{c}{ Interstitial } \\
& $V_{2}$ & $V_{3}$ & $S V$ & $V_{4}^{\prime}$ & & $I_{2}$ & $I_{2 d}$ & $I_{3}$ & $I_{4}^{\prime}$ \\
\hline$V_{2}$ & 0.9987098 & 0.0000154 & 0.0012747 & 0 & $I_{2}$ & 0.9977724 & 0.0000890 & 0.0021340 & 0.0000046 \\
$V_{3}$ & 0.0000175 & 0.9995009 & 0.0004666 & 0.0000150 & $I_{2 d}$ & 0.0051196 & 0.9947921 & 0.0000883 & 0 \\
$S V$ & 0.0009514 & 0.0000255 & 0.9990231 & 0. & $I_{3}$ & 0.0007948 & 0.0000007116 & 0.9989618 & 0.0002426 \\
$V_{4}^{\prime}$ & 0. & 0.0048446 & 0 & 0.9951554 & $I_{4}^{\prime}$ & 0.0000450 & 0.0000064 & 0.0054687 & 0.9944798 \\
\hline$\tilde{t}_{s}$ & 0.4068799 & 0.0420110 & 0.5509791 & 0.0001300 & $\tilde{t}_{s}$ & 0.2618633 & 0.0046109 & 0.7024319 & 0.0310939 \\
$\Delta E$ & $0.1044(6)$ & $0.1046(6)$ & $0.1040(6)$ & $0.1086(6)$ & $\Delta E$ & $0.0798(6)$ & $0.0799(6)$ & $0.0790(6)$ & $0.0829(6)$ \\
\hline \hline
\end{tabular}

that our results may have important bearing on experimental works involving interfaces and solid surfaces.

\section{Acknowledgments}

This research was supported by CNPq, CAPES, FAPESP and FUNAPE-UFG. Luciano da F. Costa thanks FAPESP (05/00587-5) and CNPq (308231/03-1) for financial support. The authors thank professor G.-Q. Hai for useful discussions.
* Permanent address: Departamento de Matemática, UFMT, Campus do Médio Araguaia 78698-000, Pontal do Araguaia, MT, Brazil.

1 A. Pertsinidis and X. S. Ling, Nature 413, 147 (2001); A. Pertsinidis and X. S. Ling, Phys. Rev. Lett. 87, 098303 (2001).

2 A. P. Gast and W. B. Russel, Phys. Today 51, 24 (1998).

3 A. Pertsinidis and X. S. Ling, New Journal of Physics 7, $33(2005)$

4 E. Kim and M. H. Chan, Nature 407, 225 (2004); E. Kim and M. H. Chan, Science 305, 1941 (2004).

5 S. A. Khairallah and D. M. Ceperley, Phys. Rev. Lett. 95, 185301 (2005); D. M. Ceperley and B. Bernu, Phys. Rev. Lett. 93155303 (2004).

6 D. S. Fisher, B. I. Halperin and R. Morf, Phys. Rev. B 20, 4692 (1979).

7 E. Cockayne and V. Elser, Phys. Rev. 43, 623 (1989).

8 L. Cândido, P. Phillips and D. M. Ceperley, Phys. Rev. Lett. 86, 492 (2001).
9 S. Jain and D. R. Nelson, Phys. Rev. E. 61, 1599 (2000).

10 E. Frey, D. R. Nelson and D. S. Fisher, Phys. Rev. B. 49, 9723 (1994).

11 A.A. Maradudin, Theoretical and Experimental Aspects of the Effects of Point Defects and Disorders on the Vibrations of Crystals, (Academic Press, New York/London, 1966).

12 D. Hone, J. Phys. (Paris), Colloq. 46, C3-21 (1985).

13 S. Alexander, P. M. Chaikin, P. Grant, G. J. Morales, P. Pincus, and D. Hone, J. Chem. Phys. 80, 5776 (1984).

14 F. M. Peeters and X. Wu, Phys. Rev. A 35, 31091987.

15 A. Libál, C. Reichhardt and C.J Olson Reichhardt, cond-mat/0609676 (2006).

16 H.J.C Berendsen, J.P.M. Postma, W.F. van Gusteren, A. DiNota, J. H. Haak, J. Chem. Phys. 81, 3684 (2006).

17 Stochastic geometry and its applications, D. Stoyan, W. S. Kendall and J. Mecke, John Wiley and Sons, 1995, Chichester. 